\title{
Quantifying electric vehicle battery degradation from driving vs. vehicle-to-grid services
}

\author{
Dai Wang, Jonathan Coignard, Teng Zeng, Cong Zhang, Samveg Saxena* \\ Lawrence Berkeley National Laboratory \\ 1 Cyclotron Rd., MS90R1121B, Berkeley, CA 94720
}

\begin{abstract}
The risk of accelerated electric vehicle battery degradation is commonly cited as a concern inhibiting the implementation of vehicle-to-grid (V2G) technology. However, little quantitative evidence exists in prior literature to refute or substantiate these concerns for different grid services that vehicles may offer. In this paper, a methodology is proposed to quantify electric vehicle (EV) battery degradation from driving only vs. driving and several vehicle-grid services, based on a semi-empirical lithium-ion battery capacity fade model. A detailed EV battery pack thermal model and EV powertrain model are utilized to capture the time-varying battery temperature and working parameters including current, internal resistance and state-of-charge (SOC), while an $\mathrm{EV}$ is driving and offering various grid services. We use the proposed method to simulate the battery degradation impacts from multiple vehicle-grid services including peak load shaving, frequency regulation and net load shaping. The degradation impact of these grid services is compared against baseline cases for driving and uncontrolled charging only, for several different cases of vehicle itineraries, driving distances, and climate conditions. Ten-year degradation simulation results show that frequency regulation and peak load shaving at power rates typical for vehicle charging and discharging will not significantly accelerate battery degradation in comparison to the degradation incurred from driving and calendar aging.
\end{abstract}

Keywords: electric vehicle, battery degradation, vehicle-to-grid, powertrain

\footnotetext{
${ }^{*}$ Corresponding author

Email address: samveg@berkeley . edu (Samveg Saxena)
}

Preprint submitted to Journal of $\mathrm{BT}_{E} X$ Templates

September 12, 2016 


\section{Introduction}

Electrification of transportation is commonly believed as a promising path to meet climate goals around the world [1], and prior studies have established the ability for today's commercially available EVs to meet the daily travel needs of the vast majority of drivers [2]. Moreover, if EVs can feed energy back to the grid to provide "vehicle-togrid (V2G)" services, it will bring multiple benefits to different stakeholders including both power systems and EV owners. These benefits might include frequency regulation, peak load shaving, enhancing the integration of solar and wind energy, and providing distributed energy resources to deal with unexpected outages [3]. The authors in [4] provided quantitative methods to estimate the cost and revenue of implementing V2G in four major electricity markets. The results showed that implementing V2G can increase the reliability of power grids, reduce system costs, and lower the prices of storage devices and reserves for renewable energy integration. The authors in [5] proposed a V2G algorithm to optimize the scheduling of energy, regulation and spinning reserves based on the Electric Reliability Council of Texas system. The simulation results demonstrated that $\mathrm{V} 2 \mathrm{G}$ can bring substantial benefits to both aggregators and customers. In [6, 7, 8], the authors showed that the controlled charging of EVs can increase the use of renewable energies in both distribution and transmission levels by mitigating the intermittency of solar and wind power generation.

Despite these benefits, the concern on battery degradation incurred by V2G services is one of the major hurdles to its widespread deployment [9]. Batteries will lose irreversible capacity with cycling and calendar aging. The battery pack has to be replaced, if it degrades to sufficient levels. It is traditionally assumed that batteries will reach their end-of-life (EOL) once they lose $20-30 \%$ of their original storage capacity [10]. Therefore, a quantitative methodology to estimate the EV battery degradation is the basis of evaluating the financial and technical feasibility of V2G services.

Many researchers have studied the degradation mechanisms of lithium-ion batteries which are widely used in today's EVs [11]. Capacity loss may be caused by irreversible electrochemical side-reactions(e.g. electrolyte reduction). It can also result from the 

degradation mechanisms [12]. Based on these prior studies, it is generally understood that capacity loss is related to time, temperature, discharge rate and depth of discharge (DOD). Different battery degradation models have been proposed based on several aging mechanisms including parasitic side reactions [13], solid electrolyte interphase load shaping for renewable integration, are simulated under various travel and charging itineraries, with different charger levels, and at different climate conditions.

The reminder of the paper is organized as follows. Section 2 stresses the specific objectives of this paper. The methodology is presented in Section 3. In Section 4, 60

(SEI) formation [14], and resistance increase [15]. Characterization of battery degradation from driving or grid services requires a huge experimental matrix to cover all combinations of different factors, and acquiring the test data to individually isolate and quantify each degradation mechanism is intractable.

Furthermore, evaluating EV battery degradation is even more complex due to the variability of EV battery working conditions. Battery temperature is influenced by multiple factors including solar radiation, environmental temperature, heat generated when it is charging or discharging, and the battery's thermal management system. In addition, DOD, current input/output and heat generated by the battery are determined by EV driving patterns, like trip distance and frequencies, acceleration, speed, road grade, etc. The authors in [16] presented mathematical correlations of battery wear for different conditions of charging/discharging, ambient temperature, DOD, but they did not extend their work to consider the influences of driving patterns on battery degradation. The authors in [17] quantified the impact on battery degradation while providing bulk energy and ancillary services. However, battery working temperature is assumed to have a constant value which is insufficient given that prior literature [11, 12, 13, 14] has emphasized the importance of temperature upon battery degradation.

To the best of our knowledge, this paper is the first work to present a methodology to quantify EV battery degradation from driving vs. V2G services, incorporating a detailed thermal model, travel patterns and EV powertrain model. Battery degradation caused by multiple V2G services including emergency DR, frequency regulation and

we explain the V2G services simulated in this paper. Simulation results are shown in 
Section 5. Conclusions and future work are in Section 6.

\section{Specific objectives}

Vehicle grid integration (VGI) presents a tremendous opportunity to benefit both the electricity grid and the transportation sector, yet concerns about accelerated battery degradation in vehicles that provide grid services remains an obstacle. The lack of a quantitative evaluation method for EV battery degradation amplifies people's anxiety on V2G cost and hinders stakeholders to price V2G services. Therefore, this study aims to quantify the magnitude of battery degradation that is incurred from normal driving and uncontrolled charging of vehicles versus degradation in vehicles that are driving plus offering grid services. In order to comprehensively examine the validity of concerns about battery degradation from VGI, we quantify battery degradation for several different grid services, under various travel and charging itineraries, and at different ambient temperature and solar radiation levels. This work will also provide reference for pricing or making compensation policies for different $\mathrm{V} 2 \mathrm{G}$ services.

\section{Methodology}

\subsection{Battery capacity fade model}

Lithium-ion batteries slowly lose storage capacity with calendar aging and cycling. These capacity losses mainly result from lithium inventory loss due to irreversible electrochemical side-reactions [18]. There are often two distinct, but inter-related ways that are used to measure the life of lithium-ion batteries: calendar life and cycle life. Calendar life represents how long the battery might be expected to last in terms of calendar years, while cycle life is typically expressed in terms of number of charge and discharge cycles. A recent research [19] developed a semi-empirical model to calculate the capacity losses caused by both cycling and calendar aging for a particular lithium ion chemistry. It takes into account four important experimental parameters including temperature, time, depth of discharge (DOD) and charge/discharge rate. Moreover, this model decouples the capacity loss caused by cycling and calendar aging. Total capacity loss can be calculated by adding them together during any time horizon. 
Calendar aging is strongly affected by temperature $(T)$ and time $(t)$. Capacity loss of a battery cell induced by calendar aging is expressed as [19]:

$$
Q_{\text {loss }}^{\text {calendar }}=f e^{-E_{a} / R T} t^{1 / 2}
$$

where $Q_{\text {loss }}^{\text {calendar }}$ is the percentage of capacity loss induced by calendar aging, $f$ is the 90 pre-exponential factor, which is 14876 day $^{-1 / 2} . E_{a}$ is the activation energy, which is $24.5 \mathrm{~kJ} \cdot \mathrm{mol}^{-1} . R$ is the gas constant, which is $8.314 \mathrm{~J} \cdot \mathrm{mol}^{-1} \mathrm{~K}^{-1} . T$ is the absolute temperature in $\mathrm{K}$.

Cycle life loss is related to temperature, charge/discharge rate and energy throughput. The effects of discharge rates can be captured by an exponential function. Therefore, capacity loss of a battery cell induced by cycling is expressed as [19]:

$$
\begin{aligned}
& Q_{\text {loss }}^{\text {cycle }}=B_{1} \cdot e^{B_{2} \cdot I_{\text {rate }}} \cdot A_{h} \\
& B_{1}=a \cdot T^{2}+b \cdot T+c \\
& B_{2}=d \cdot T+e
\end{aligned}
$$

where $Q_{\text {loss }}^{\text {cycle }}$ is the percentage of capacity loss induced by cycling. Both the preexponential factor $B_{1}$ and exponential factor $B_{2}$ are functions of temperature. The values of $a, b, c$ are $8.89 \times 10^{-6} \mathrm{Ah}^{-1} \mathrm{~K}^{-2},-0.0053 \mathrm{Ah}^{-1} \mathrm{~K}^{-1}, 0.7871 \mathrm{Ah}^{-1}$, respectively. $I_{\text {rate }}$ is the charge/discharge rate in C-rate. $A_{h}$ is the Ah-throughput, which is expressed as $A_{h}=($ cycle number $) \times(D O D) \times($ full cell capacity $)$. Alternatively, $A_{h}$ can be expressed as the cumulative charge throughput, i.e. the integral of $I_{\text {rate }}$. At each C-rate, Ah-throughput is directly proportional to time.

Combining (1) and (2), the general function of battery capacity loss is:

$$
Q_{\text {loss }}^{\text {total }}=\left(a \cdot T^{2}+b \cdot T+c\right) e^{(d \cdot T+e) \cdot I_{\text {rate }}} \cdot A_{h}+f \cdot e^{-E_{a} / R T} \cdot t^{1 / 2}
$$

It should be noted these equations are obtained from the experiments on the commercially available 18650 cylindrical cells [19] which have been considered as a viable candidate for vehicular applications. Other batteries can degrade differently.

From (1) - 3], we can see that battery capacity loss is a continuous function of temperature, current and time. It is convenient to use (3) to calculate the battery degradation at a fixed working condition. However, the current and temperature of EV battery 
pack keep changing when the vehicle is driving. In order to capture the degradation under a practical working condition, we need to discretize both calendar life and cycle life equations. Discretizing the calendar life loss equation (1), the incremental degradation from calendar aging at time $\hat{t}$ is

$$
\Delta Q_{\text {loss }}^{\text {calendar }}(\hat{t})=\left.\frac{\partial Q_{\text {loss }}^{\text {calendar }}(t)}{\partial t}\right|_{t=\hat{t}} \Delta t=0.5 A e^{-E_{a} / R T} t^{-0.5} \Delta t
$$

Ah-throughput can be interpreted as total energy in Ah which is output from the battery. For a constant charging rate which lasts for time $\Delta t$, it can be expressed as

$$
A_{h}(\Delta t)=I_{\text {rate }} \times \Delta t
$$

Discretizing the cycle life loss equation (2), the incremental degradation from cycling at time $\hat{t}$ is:

$$
\Delta Q_{\text {loss }}^{\text {cycle }}(\hat{t})=\left.\frac{\partial Q_{\text {loss }}^{\text {cycle }}(t)}{\partial t}\right|_{t=\hat{t}} \Delta t=B_{1} \cdot e^{B_{2} \cdot I_{\text {rate }}} \cdot I_{\text {rate }} \Delta t
$$

Thus, the total capacity loss $Q_{\text {loss }}$ of battery by time $t$ is the sum of all capacity losses at all contributing time steps

$$
Q_{\text {loss }}(t)=\sum_{\hat{t}=0}^{t}\left(Q_{\text {loss }}^{\text {cycle }}(\hat{t})+Q_{\text {loss }}^{\text {calendar }}(\hat{t})\right)
$$

\subsection{EV battery temperature model}

From the battery capacity fade model, we can see that temperature is one of the dominant factors. Therefore, it is necessary to provide an accurate estimate of battery temperature. A car-level lumped capacitance thermal network approach [20] is utilized for calculating both cabin heating, ventilation and air conditioning (HVAC) loads and battery temperature. Equations $(8)$ - (9) define the lumped capacitance thermal network of the battery and its environment.

$$
\begin{aligned}
& M_{c} \frac{d T_{c}}{d t}=K_{a c}\left(T_{a}-T_{c}\right)+K_{b c}\left(T_{b}-T_{c}\right)+q_{r a d}+q_{h v a c} \\
& M_{b} \frac{d T_{b}}{d t}=K_{a b}\left(T_{a}-T_{b}\right)+K_{b c}\left(T_{c}-T_{b}\right)+q_{b t m s}+Q
\end{aligned}
$$


where $T_{a}$ is the ambient temperature, $T_{b}$ is the battery temperature, $T_{c}$ is the cabin temperature. $K_{a b}$ is the effective heat transfer coefficient between ambient and battery; $K_{a c}$ is the effective heat transfer coefficient between ambient and cabin; $K_{b c}$ is the effective heat transfer coefficient between battery and cabin. $M_{c}$ is the thermal mass of vehicle cabin; $M_{b}$ is the thermal mass of battery. $q_{r a d}$ is the solar radiance, which depends on time and location. $q_{\text {hvac }}$ is the heat added to or removed from the cabin by the HVAC system. $q_{b t m s}$ is the heat added to or removed by the battery thermal management system. $Q$ is the heat generated by the battery. Ambient temperature and solar radiation data are sourced from historical databases. The values of parameters are calculated to fit the response of this model to test data [20]. They are listed in Table. 1]

Table 1: Values of parameters in the EV thermal model.

\begin{tabular}{ll}
\hline Parameter & Value \\
\hline$K_{a b}$ & $4.343 \mathrm{WK}^{-1}$ \\
& $22.6 \mathrm{WK}^{-1}\left(T_{c}<T_{a}\right)$ \\
$K_{a c}$ & $22.6+1500 \mathrm{WK}^{-1}\left(T_{c}>T_{a}\right.$, key-on $)$ \\
& $3.468 \mathrm{WK}^{-1}$ \\
$K_{b c}$ & $182,000 \mathrm{JK}^{-1}$ \\
$M_{b}$ & $101,771 \mathrm{JK}^{-1}$ \\
$M_{c}$ & $+4 \mathrm{~kW}\left(T_{c}<292\right.$, key-on $)$ \\
$q_{h v a c}$ & $-4.5 \mathrm{~kW}\left(T_{c}>297\right.$, key-on $)$ \\
\hline
\end{tabular}

How to calculate $Q$ is a key question before using this temperature model. The heat generated from the battery is:

$$
Q=I^{2} \times R
$$

where $I$ is the current input/output, $R$ is the internal resistance. Intuitively, the current is related to speed and acceleration. High speed and acceleration usually require high current output to power the vehicle. Therefore, we should consider the EV powertrain model, which can provide the detailed battery parameters when it is in use for any driving conditions. 


\subsection{Acquisition of battery working parameters}

As discussed above, we need to acquire the time-resolved current and internal resistance in order to calculate the heat generated from the battery while it is cycling for driving or V2G services. In addition, current input/output and real-time SOC are also required for calculating cycle life loss as shown in (3).

A simulation tool called "V2G-Sim", which we developed previously [2, 9, 21, 22], is applied in this study to obtain detailed EV information and battery working parameters. In this work, V2G-Sim is provided with travel itinerary input data from the National Household Travel Survey (NHTS) [23], which contains 159,844 representative samples of weekday and weekend vehicle usage for drivers in the United States. It conveys the detailed information on each trip, including trip distances, start and end times, and types of locations (e.g. home, work, etc.). Table. 2 shows an example of the travel itinerary information input into V2G-Sim from the NHTS dataset.

Table 2: An example of travel itinerary information provided to V2G-Sim

\begin{tabular}{ccccc}
\hline Start time & End time & Event type & Distance/charger level & Location \\
\hline $0: 00$ & $8: 45$ & Plugged in & L1 & Home \\
$8: 45$ & $9: 45$ & Driving & $39.6 \mathrm{mi}$ & N/A \\
$9: 45$ & $16: 30$ & Parked & L2 & Work \\
$16: 30$ & $17: 00$ & Driving & $5.0 \mathrm{mi}$ & N/A \\
$17: 00$ & $17: 30$ & Parked & N/A & Restaurant \\
17:30 & $18: 00$ & Driving & $5.0 \mathrm{mi}$ & N/A \\
$18: 00$ & $21: 00$ & Parked & L2 & Work \\
$21: 00$ & $22: 00$ & Driving & $39.6 \mathrm{mi}$ & N/A \\
$22: 00$ & $0: 00$ & Plugged in & L1 & Home \\
\hline
\end{tabular}
in Table. 2. V2G-Sim calls relevant sub-models which tracks energy consumption and other parameters in the vehicle powertrain. The term powertrain describes the main components that generate power and deliver it to the road surface. This includes the transmission, engine, differentials, drive shafts, drive wheels, etc. A detailed pow- 
the energy consumption of any vehicle make/model on any trip-specific drive cycle (including terrain considerations), and with any level of ancillary power loading (e.g. from a vehicle's HVAC system). In this paper, we use the powertrain specifications for a "Nissan Leaf" [9].

155 $1.4 \mathrm{~kW}$, L2 charger at $7.2 \mathrm{~kW}$, or super charger at $120 \mathrm{~kW}$ ), charging sub-models are used to calculate the power transfer from the electricity grid to that vehicle. When the vehicle is driving, a trip-specific drive cycle profile and the terrain during the trip will be input into the detailed powertrain simulation. In this paper, we generate the drive cycles from the US Environmental Protection Agency (EPA) standard drive cycles for city (UDDS), highway (HWFET), and aggressive driving (US06), as shown in Fig. S1 in the supplementary material, however the models can be provided with any real world drive cycle.

Since the battery degradation model in Section 3.1 is for individual cell, we need 165 and SOC in the battery pack is homogeneous. That means the SOC and temperature of all battery cells are the same as the pack-level values. In this paper, we use the configurations of "Nissan Leaf" battery package which has 48 modules in series. Each module is formed by connecting 4 cells in a 2-series, 2-parallel formation. Therefore, the current through each cell is half of the current input/output from the whole battery package.

In this manner, each vehicle's SOC, current and internal resistance are computed on a second-by-second basis.

\subsection{Simulation flow}

175

After obtaining temperature data and other working parameters, we can calculate the battery degradation according to (4) - (7). As shown in Fig. 1, given EV itineraries and V2G strategies, V2G-Sim will specify all driving/charging/discharging/idle events and assign a drive cycle to each trip. After setting charging/discharging parameters, all information will be imported into the detailed powertrain model. Then, we can acquire 
SOC, current, and internal resistance. Values of internal resistance and current will further go to the temperature module where solar radiation and ambient temperature are sourced from historical data. Battery temperature will be used to calculate calendar life loss according to (4). Current, SOC and temperature will be used to calculate cycle life loss according to (6). Finally, we add them together and get the total capacity loss.

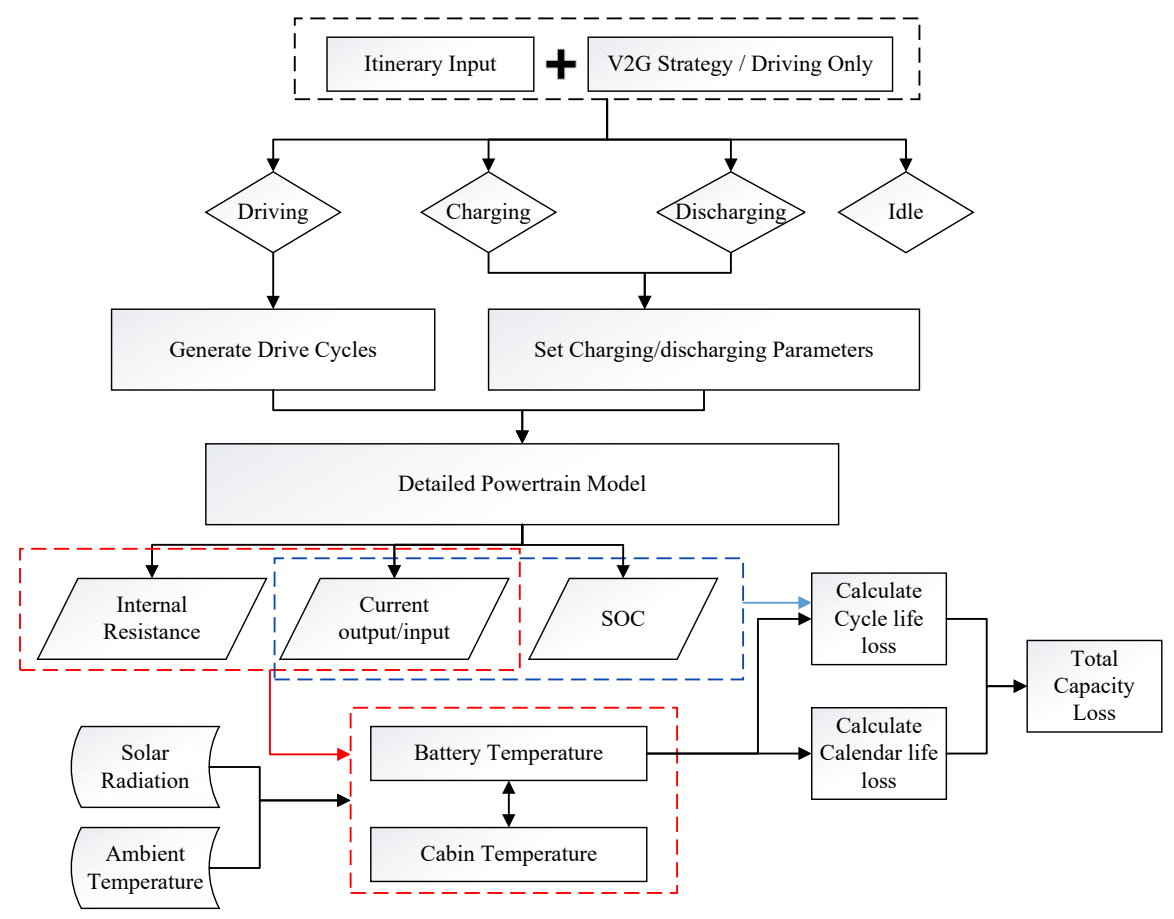

Figure 1: The whole framework of quantifying EV battery degradation.

It should be noted that the proposed method is ex-post and simulation-based. Given a specific strategy and itinerary, we can calculate battery degradation second-by-second. If the replacement price of the EV battery is known, we can further translate the irreversible capacity loss to the EV cost from providing grid services, according to specific End-of-Life criterion of EV battery. The cost of battery degradation for an individual $\mathrm{EV} C_{e v}$ for a grid service event will be:

$$
C_{e v}=\frac{Q_{l o s s}^{g s} \%}{\eta \%} \cdot P_{b}
$$


which is commonly (though arbitrary in nature [9]) set to be between 20-30\%. If the battery loses its capacity by $\eta \%$, it reaches the end of life. $P_{b}$ is the price of the whole battery pack (e.g. the cost to replace a pack).

\section{Simulated grid services}

In this paper, we explore the EV battery degradation in four different cases. They are uncontrolled charging, peak load shaving, frequency regulation and net load shaping. In all these cases, we ensure that the grid services offered by each vehicle does not compromise the mobility needs of each driver.

\subsection{Driving and uncontrolled charging (Base case)}

In the base case, all EVs are used only for driving. They do not provide any grid services. It is assumed that EVs can charge either at home or workplace. Once they arrive at home or workplace, they start to charge immediately. The charging process will not end until the battery pack is full or the EV has to start the next trip. We show the weekday charging load of 17805 vehicles in California from NHTS dataset in Fig.

\subsection{Peak load shaving}

Peak load spikes endanger the safety and reliability of electric power networks. Shaving the load peak can not only alleviate the shortage of supply and strengthen the stability of power systems, but also enable a lower transmission capacity, thereby reducing the capital cost of transmission infrastructure. During peak load times, curtailment of EV charging load can provide a quick-responsive resource. If V2G is enabled, EVs can also feed energy back to the grid during these periods. Examples of the 
charging load profiles in both V1G and V2G cases are shown in Fig. S3 in the supplementary material. EVs shift their charging demand from 17:00-21:00 to other time periods. Negative charging load means the vehicles are discharging at that time. Peak load shaving may be required when extreme hot or cold weather results in the power demand peak. In addition, since the electricity price is usually higher during the load peak time, people may be also interested in permanent load shifting to do arbitrage.

\subsection{Frequency regulation}

Frequency regulation is a service that corrects for short-term changes in electricity use that might affect the stability of the power system. It helps match generation and demand and adjusts generation output to maintain the desired electrical frequency for the grid to function normally. EVs can respond to the frequency regulation signal and act as demand-side regulating resources. It is often believed that frequency regulation is one of the most lucrative V2G services. The pilot project led by University of Delaware demonstrated that Electric BMW Minis can earn approximately $\$ 100$ per month per car by following PJM frequency regulation signals [27].

In the PJM regulation market, a fast response regulation signal (RegD) is separated from the traditional regulation signal (RegA). Compared with RegA signal, RegD is derived with a high-pass filter, thereby having much faster movement. An example of PJM regulation signals including RegA and RegD is shown in Fig. S4 in the supplementary material. Since EV batteries are able to ramp from zero to full power output within a very short period, they can provide the fast response regulation service which is more lucrative. In addition, RegD signal is symmetrical, which requires zero net energy over a 15-min time period. This favorable characteristics reduces the amount of obligated reserved energy in the EV battery pack. In this paper, we simulate the scenario that vehicles can precisely follow the RegD signal.

\subsection{Net load shaping}

Since EVs can be either flexible loads or power sources, they have the potential to enable high penetration of renewables by balancing loads with intermittent generation. In this paper, we study the EV battery degradation from performing net load shaping, 
which aims to mitigate the fluctuation of net load. A distributed optimal charging algorithm [28] has been built into V2G-Sim. The objective $[12]$ is to minimize the variance of net load.

$$
\min _{u} \sum_{t=1}^{T}\left(D^{t}+\sum_{n=1}^{N} u_{n}^{t}\right)^{2}
$$

where $N$ is the number of EVs; $T$ is the time horizon; $u_{n}^{t}$ is the charging rate of EV $n$ at time $t ; D^{t}$ is the net load at time $t$ (total load minus renewable generation). $u_{n}^{t}$ is the decision variable. An EV is charging if $u_{n}^{t}>0$, while it is discharging if $u_{n}^{t}<0$. There are also power and energy constraints, which guarantee the charging/discharging power does not exceed the power limits and all EVs have enough energy for the drivers' desired travels.

A load shaping case for the so-called California "Duck Curve" is given in Fig. S5 in the supplementary material. Due to the features of wind and solar generation, the net load reaches a deep valley in the daytime and a high peak at 19:00. The short and steep ramps require the operator to bring on or shut down generation resources to meet an increasing or decreasing electricity demand quickly, over a short period of time. Uncontrolled charging even makes the situation worse. If V1G control is performed, vehicles opt for charging during the daytime valley, but it cannot help lower the load peak. However, in V2G case, EVs will charge during valley time and discharge during peak time. It can be seen that the fluctuation of net load is significantly mitigated.

\section{Results and Discussions}

In this section, we quantify the EV battery degradation from each grid service defined in Section 4. Parametric simulations are conducted for different travel and charging itineraries, different grid service durations, different charging/discharging rates, and different ambient temperature and solar radiation levels.

\subsection{Driving + uncontrolled charging}

We firstly study the battery capacity loss from normal driving and uncontrolled charging. To get the general results which can cover different itineraries, drive cycles and travel distances, we randomly pick up 100 vehicles from NHTS dataset and assume 
all EVs repeat the same daily travel itineraries for 10 years. EVs can charge either at home or workplace. Once they arrive at home or workplace, they start to charge immediately. The charging process will not end until the battery pack is full or the EV has to start the next trip. We assume all EVs use level charger $(1.44 \mathrm{~kW})$ at home and level charger (7.2kW) at work locations. Ambient temperature [25] and solar radiation [26] data from Phoenix, San Francisco and Minneapolis, which represent three typical climates, are used to show the battery degradation caused by cycling and calendar aging. We can see from Fig. 2 (a) that the degradation incurred by calendar aging is fast in the beginning and slows down as time progresses, since it follows a time $e^{0.5}$ relationship according to (1). Calendar aging is also related to the battery temperature. It can be seen that the battery calendar aging in Minneapolis is minimal because of the cold ambient temperature. However, the battery degradation caused by cycling in Minneapolis is much more than that in other cities. Fig. 2(b) shows that the 10-year battery capacity loss caused by cycling in Minneapolis is over 25\%. It should be noted that we do not consider an active heating system here. If the heating system can warm up the battery before cycling, the degradation in cold cities will be significantly reduced, however this will cause a larger penalty in the EV travel range. After understanding the influences of temperature and solar radiation, we use the climate data of San Francisco to perform the sensitive analysis for other factors in the following simulations.

For the "Driving + uncontrolled charging" case, we also simulate the 10-year average battery degradation of $100 \mathrm{EVs}$ in four different scenarios of charging, including level $1(1.44 \mathrm{~kW})$ or level $2(7.2 \mathrm{~kW})$ charging at home and work locations. Fig. 3 shows that in the case of driving only with uncontrolled charging, typical EV batteries lose around $31 \%$ capacity after ten years. Using an L2 charger has only minor effects on the battery degradation compared to using L1 charger. In the following simulations, the battery degradation curve with $\mathrm{L} 1$ charger at home and L2 charger at work location will be used as the baseline to evaluate the impacts of different grid services.

\subsection{Peak load shaving}

Next we simulate the battery degradation caused by providing peak load shaving, which is defined in Section 4.2. Both V1G and V2G scenarios are considered. Sensi- 


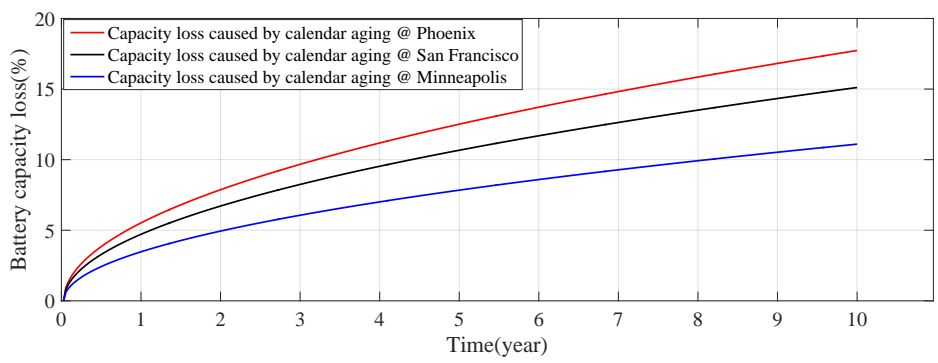

(a)

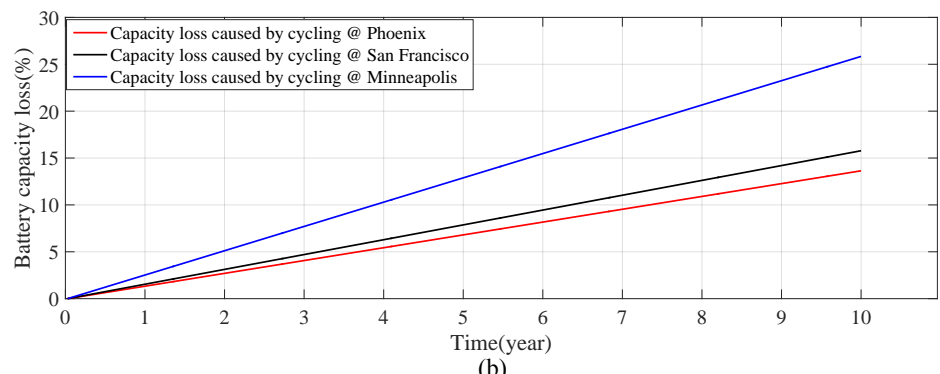

(b)

Figure 2: Capacity loss incurred by (a) calendar aging and (b) cycling, in 3 typical climate cities. The results are the average values over 100 vehicles. Calendar life loss is fast in the beginning and slows down as time progresses. Although low temperature is helpful to slowing down the calendar aging, it will result in more cycle life loss.

tivity analysis on the time length of peak shaving event and charging/discharging rate is conducted. Fig. 4 (a) shows the average degradation of 100 vehicles by performing the peak load shaving from 19:00-21:00 every day for ten years. In the V1G case, all vehicles avoid charging during this period. Different charger combinations only make a slight difference on the battery degradation. In the V2G scenario, the grid service results in more capacity loss than that in V1G scenario. Since most vehicles are at home from 19:00-21:00, using an L2 charger at home in the V2G scenario will greatly increase the energy fed back to the grid, thereby accelerating the battery degradation. It can be seen that the 10-year capacity loss by using an L2 charger at home is over $45 \%$, which is $9 \%$ more than that by using an $\mathrm{L} 1$ charger at home.

Fig. 4(b) shows the battery degradation with different peak shaving hours. Here we assume L1 charger is used at home and L2 charger is used at work location. If vehicles 


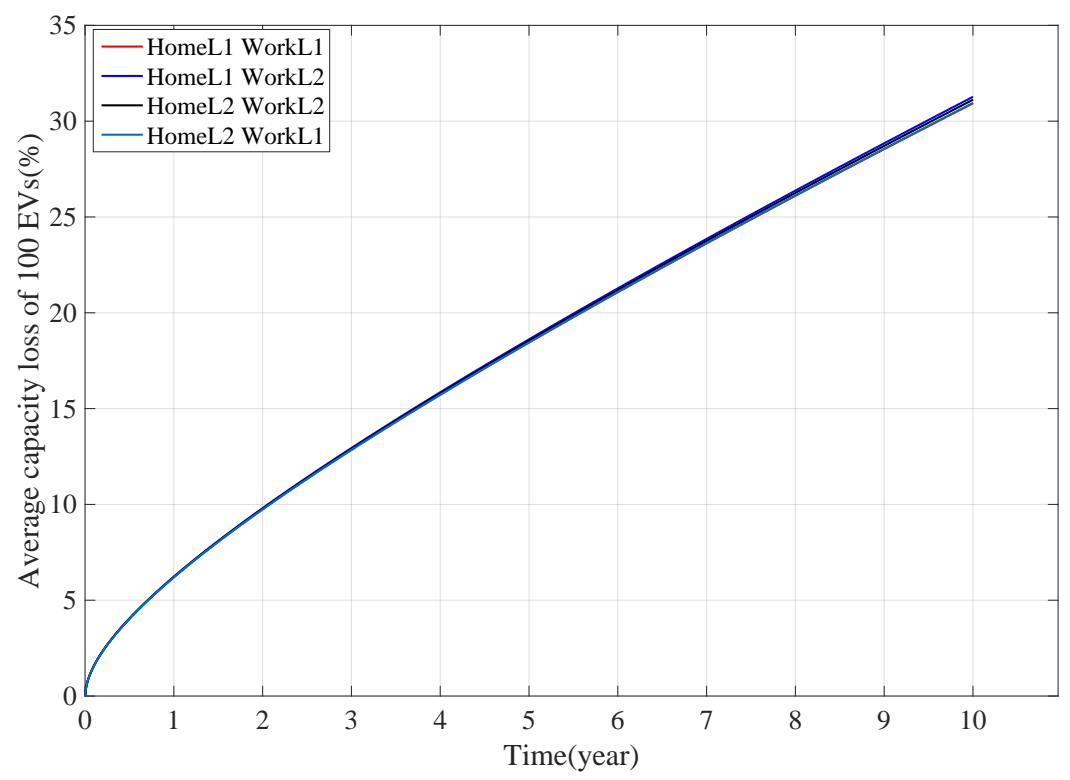

Figure 3: Average battery degradation of $100 \mathrm{EVs}$ with different charger availability in driving + uncontrolled charging case. Using an L2 charger just has minor impacts on the battery degradation. Capacity loss after ten years is about $31 \%$.

perform permanent peak load shaving every day for ten years, the total capacity losses by performing one to four hour services are $34.2 \%, 37.0 \%, 38.8 \%, 41.1 \%$, respectively.

The above results show the capacity losses if EVs perform permanent peak shaving every day. It also helps people who are interested in utilizing EV for energy arbitrage to understand the magnitude of battery degradation from permanent load shifting. However, based on the emergency demand response programs of utility companies [29], peak load shaving may just be called 20 times per year in emergency events. Here we choose the 20 hottest days of a year and calculate the battery capacity losses if EVs provide peak load shaving (V2G) from 19:00-21:00 on these days. As shown in Table. 3 , the $\mathrm{V} 2 \mathrm{G}$ emergency peak load shaving increases the capacity losses by $0.38 \%$ with L1 charger at home and $0.82 \%$ with L2 charger at home after ten years, compared to the base case. Average capacity losses from each peak shaving event are $0.0019 \%$ and $0.0041 \%$. If the replacement price of the whole battery pack is $\$ 6000$ and $30 \%$ is the end of life, the battery degradation cost from the 2-hour emergency providing peak load 

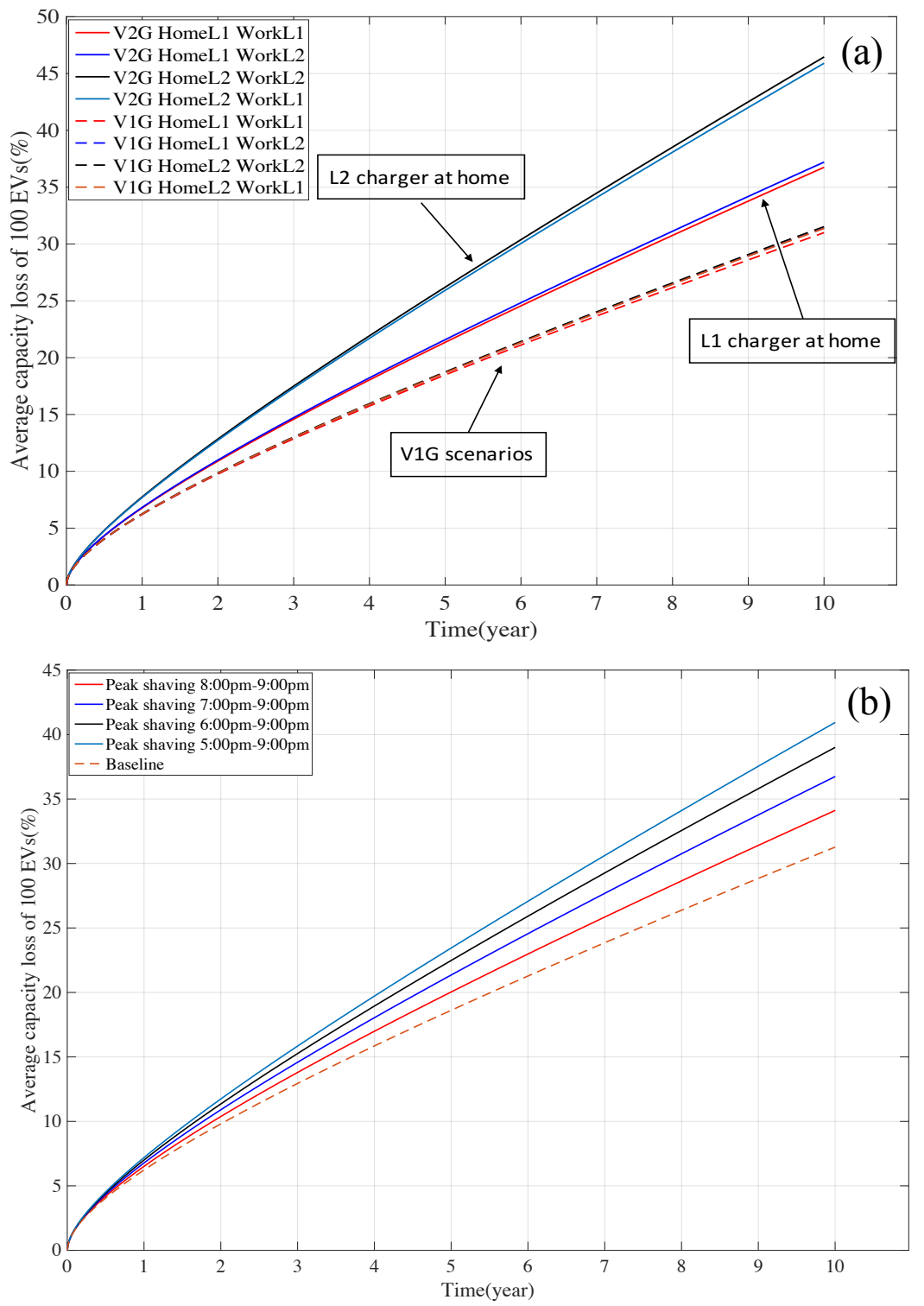

Figure 4: Average 10-year battery degradation of $100 \mathrm{EVs}$ for providing peak load shaving with (a) different charger availability; (b) different peak shaving hours.

shaving (V2G) would be $\$ 0.38$ using L1 charger at home and $\$ 0.82$ using L2 charger at home. These results provide reference for pricing or making compensation policies for emergency peak load shaving. EV owners can also be aware of the monetary cost 
Table 3: Battery capacity loss if EVs provide emergency peak load shaving (V2G) from 19:00-21:00 on 20

hottest days every year for ten years. The 10-year degradation in the driving and uncontrolled charging (base case) is $31.41 \%$.

\begin{tabular}{cccc}
\hline Charger level & $\begin{array}{c}\text { Total capacity loss } \\
\text { after ten years }(\%)\end{array}$ & $\begin{array}{c}\text { Average degradation } \\
\text { from each event }(\%)\end{array}$ & $\begin{array}{c}\text { Battery cost for } \\
\text { each event (\$) }\end{array}$ \\
\hline Home L1 Work L2 & 31.79 & 0.0019 & 0.38 \\
Home L2 Work L2 & 32.23 & 0.0041 & 0.82 \\
\hline
\end{tabular}

if their EVs are used for this V2G grid service.

\subsection{Frequency regulation}

In the frequency regulation case, we also discuss the influence of charging/discharging rate and length of grid service time. Fig. 5(a) shows the 10-year average capacity loss of $100 \mathrm{EVs}$ with different charging/discharging levels. It is assumed that all EVs can accurately follow the frequency regulation signal from 19:00-21:00 and repeat for ten years. The result shows that for the V2G frequency regulation, the battery will lose $42.56 \%$ capacity if L2 charger is deployed at home, while the capacity loss will be about $35.03 \%$ if L1 charger is used at home. This compares with $31.41 \%$ capacity loss in the baseline scenario of the same vehicles simply driving and charging in an uncontrolled manner.

In the electricity market, regulation service periods are determined by the dayahead bidding and economic dispatch. However, the power economics are beyond the scope of this paper. Here we simulate four different scenarios with different regulation service hours including all day regulation, work time regulation (9:00am-5:00pm), home time regulation (5:00pm-9:00am) and 4-hour peak time regulation (5:00pm9:00pm). It can be seen from Fig. 5 (b) that all day regulation will increase 33\% capacity loss after ten years, compared with the baseline. For the work time regulation scenario, the ten-year capacity loss is $51 \%$, which is $20 \%$ more than that in the base case. Even though the work time is shorter than the home time, the work-time degradation is more due to a higher discharging rate.

It should be noted that the above results are very "extreme", because we assume 

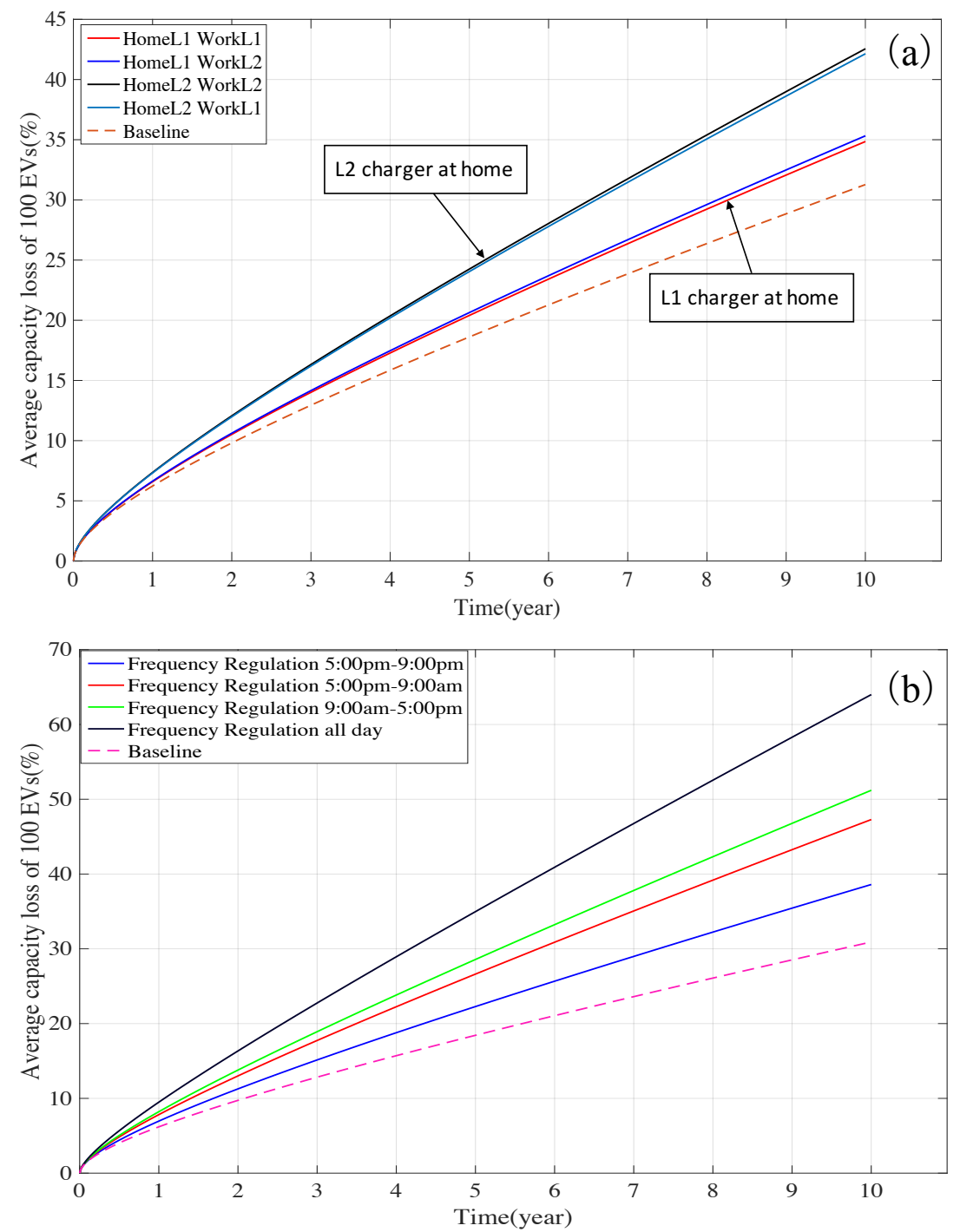

Figure 5: Average 10-year battery degradation of $100 \mathrm{EV}$ s for providing V2G frequency regulation with (a) different charger availability; (b) different regulation hours.

these EVs provide the frequency regulation service every day for ten years.It is obvious that the battery degradation will be slower if EVs are called for fewer times. In Table. 4, we show the 10-year battery capacity losses with different numbers of frequency regulation events per year. The results are the average values over $100 \mathrm{EVs}$, 
Table 4: Increase in the battery capacity loss when EVs provide the frequency regulation service (V2G) for different numbers of days every year. 10-year degradation in the driving and uncontrolled charging (base case) is $31.41 \%$. The regulation period is from 19:00 to 21:00.

\begin{tabular}{lccc}
\hline & \multicolumn{2}{c}{ 10-year battery capacity loss (\%) } \\
\cline { 3 - 4 } & & Home L1 Work L2 & Home L2 Work L2 \\
\hline \multirow{2}{*}{ Times of frequency } & 20 times & 31.62 & 31.88 \\
regulation events & 50 times & 31.93 & 32.55 \\
per year & 100 times & 32.40 & 33.73 \\
& 200 times & 33.41 & 36.01 \\
\hline Average degradation in each event $(\%)$ & 0.0010 & 0.0023 \\
\hline \multicolumn{2}{l}{ Battery cost for each event $(\$)$} & 0.2 & 0.46
\end{tabular}

and we assume the regulation period is from 19:00-21:00. It can be seen that the average capacity losses in each frequency regulation event are $0.0010 \%$ and $0.0023 \%$ with different charger availability at home or work locations. If we assume the replacement price of the whole battery pack is $\$ 6000$ and $30 \%$ is the end of life, the battery degradation cost from the 2-hour frequency regulation (V2G) would be $\$ 0.2$ using L1 charger at home and \$0.46 using L2 charger at home.

\subsection{Net load shaping}

In this case, EVs are used to mitigate the fluctuation of net load caused by the high renewable energy penetration. Fig. 6 shows the average 10-year degradation of 100 EVs in both V1G and V2G scenarios. In the V1G scenario, since all EVs only adjust their charging periods to avoid charging during peak hours, it will not cause extra degradation. However, in the V2G scenario, even using L1 charger at home will significantly accelerate the battery degradation.

To clearly illustrate what happens to an individual EV, we use FIg. 7 to show the SOC of a sample vehicle when performing this grid service. It can be seen in the V1G scenario, using L1 or L2 charger at home will not make any difference on the SOC profile. The vehicle avoids charging in the evening when the net load is high. In the V2G scenario, both SOC profiles reach two valleys. For the sake of filling the net load 


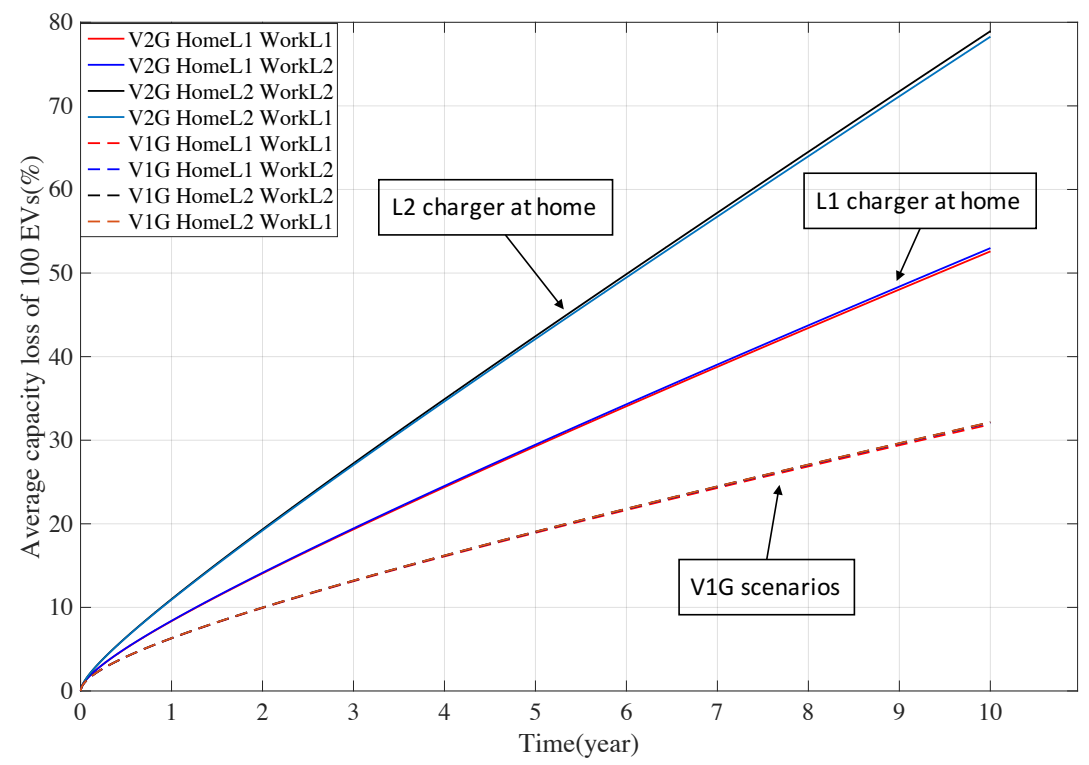

Figure 6: Average battery degradation of $100 \mathrm{EVs}$ in the net load shaping case. In V1G scenarios, using different chargers at home and work locations does not cause different degradation results. These curves are almost overlapped. In V2G scenarios, using L2 charger at home will significantly increase the battery degradation.

valley, the vehicle has to discharge from 5:00am - 9:00 am. From 6:00pm-9:00pm, the vehicle discharges in order to shave the peak. We can also see that the SOC valleys are deeper when using L2 charger at home. If L1 charger is used at home, limited by the maximum charging/discharging rate, the vehicle is not able to discharge as much as possible to make room to store the energy for the upcoming charging event. Therefore, using L1 charger at home causes less battery degradation because of less energy output from the battery.

The above results show the battery degradation if EVs provide general net load shaping service every day for ten years. However, extreme "duck curve" may only happen on a couple of days per year in reality. Here, we assume that EVs are called to perform the net load shaping service on 20 days of a year, and calculate the battery capacity losses incurred by the V2G service. As shown in Table. 5, the V2G net load shaping increases the capacity losses by $1.18 \%$ with $\mathrm{L} 1$ charger at home and $2.60 \%$ 


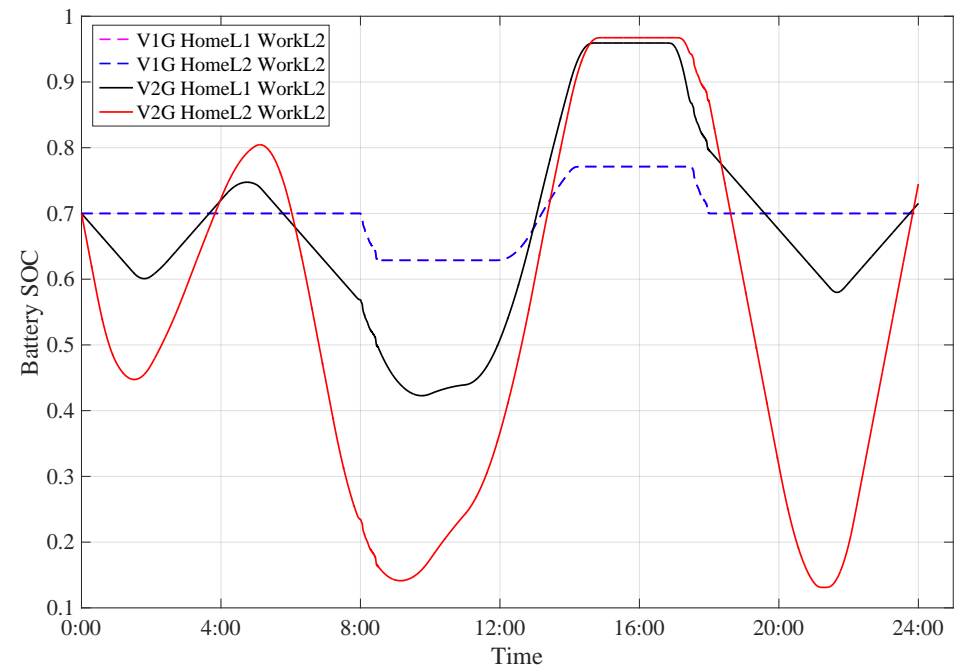

Figure 7: Battery SOC profiles of an individual EV when performing load shaping service. The SOC profiles in V1G scenarios are overlapped. The vehicle avoids charging during the load peak time. In V2G scenarios, using L2 charger at home causes more energy output from the battery. The battery keeps charging and discharging almost whole day.

with L2 charger at home after ten years, compared to the base case. Average capacity losses from each peak shaving event are $0.0059 \%$ and $0.013 \%$. If the replacement price cost from the net load shaping (V2G) for one day would be \$1.18 using L1 charger at home and $\$ 2.60$ using L2 charger at home.

\subsection{Comparisons across different grid services}

After discussing each of these grid services, we put them together and compare to the baseline. Since V1G services do not result in extra battery degradation, we show the battery capacity losses from V2G services here. The average capacity loss of 100 EVs over 10 years for different grid services are shown in Fig. 8 V2G service hours for the peak load shaving and frequency regulation are from 7:00pm-9:00pm. For the three extreme cases, each vehicle offers the chosen grid service daily for ten years. 
Table 5: Average battery capacity losses of 100 EVs if they provide the net load shaping (V2G) on 20 days per year for ten years. The 10-year degradation in the driving and uncontrolled charging (base case) is $31.41 \%$.

\begin{tabular}{cccc}
\hline Charger level & $\begin{array}{c}\text { Total capacity loss } \\
\text { after ten years }(\%)\end{array}$ & $\begin{array}{c}\text { Average degradation } \\
\text { from each event }(\%)\end{array}$ & $\begin{array}{c}\text { Battery cost for } \\
\text { each day }(\$)\end{array}$ \\
\hline Home L1 Work L2 & 32.59 & 0.0059 & 1.18 \\
Home L2 Work L2 & 34.01 & 0.013 & 2.60 \\
\hline
\end{tabular}

shaving are $3.62 \%$ and $5.60 \%$, compared with the capacity loss in the driving and uncontrolled charging case. In the net load shaping case, because EVs keep charging and discharging almost whole day, the capacity loss is much more than the base case. However, in real cases, the emergency peak load shaving and extreme "duck curve" only happen a couple of times per year. We simulate the cases that $100 \mathrm{EVs}$ provide these services 20 times per year for ten years. The results show that the V2G services only have minor impacts on the battery degradation. For the peak load shaving, frequency regulation and net load shaping, the 10-year average capacity losses are $0.38 \%, 0.21 \%$ and $1.18 \%$ more than that in the base case, respectively. If we assume $30 \%$ as the end of life, these grid services will shorten the life time of the battery by $0.25,0.19,0.51$ years.

It should be noted that we focus on quantifying the battery degradation caused by different grid services in this paper. The profits of $\mathrm{V} 2 \mathrm{G}$ for both $\mathrm{EV}$ owners and power grids, which depend on the system operation conditions and market mechanisms, are beyond the scope of this paper. In the future work, we will continue to evaluate the benefits of $\mathrm{V} 2 \mathrm{G}$ and profit allocation among different stakeholders.

\section{Conclusions}

In this paper, we present a methodology to quantify EV battery degradation caused by driving and V2G services. A semi-empirical battery degradation model is utilized to calculate battery capacity loss induced by both calendar aging and cycling. Since the temperature is a key factor which effects battery degradation, we integrate an EV 


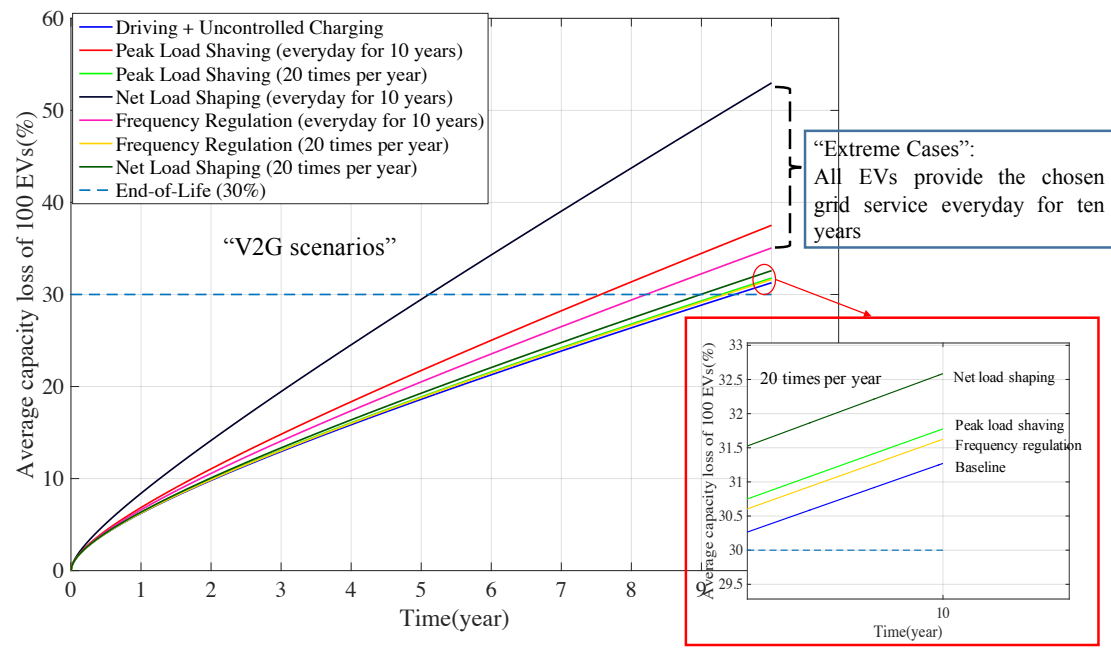

Figure 8: Average capacity losses of $100 \mathrm{EVs}$ by performing different $\mathrm{V} 2 \mathrm{G}$ services for ten years. In the extreme cases, all EVs provide the chosen grid service every day. The ten-year capacity losses from peak load shaving, frequency regulation and net load shaping increase by $3.62 \%, 5.6 \%$ and $22.6 \%$ compared to the base case, respectively. In the more realistic cases, EVs provide V2G services for 20 times per year. The ten year capacity losses increase by $0.38 \%, 0.21 \%$ and $1.18 \%$, respectively.

thermal model. Battery working parameters, including current, SOC and internal resistance, are acquired by employing the detailed EV powertrain model. Following the proposed methodology, the second by second degradation results can be obtained, with respect to time-varying working conditions of EV batteries. This methodology captures the interactions among thermal model, EV powertrain and drive cycles. Even though we use this specific semi-empirical capacity fade model in this paper, the method is not limited to this model. If there are other capacity fade models for different chemistries and battery designs, they can be also implemented into the proposed framework.

We use the proposed method to simulate the battery degradation impacts from multiple vehicle-grid services including frequency regulation, peak load shaving, net load shaping with high renewable energy penetration. The degradation impacts of these grid services are compared against the baseline case for driving plus uncontrolled charging. The simulation results lead to the following broadly applicable findings:

1. Frequency regulation and peak load shaving at power rates typical for vehicle 
charging and discharging will not significantly accelerate battery degradation in comparison to the degradation incurred from driving and calendar aging. Even in the "extreme" cases in which we assume all EVs provide grid services from 7:00pm-9:00pm every day for ten years, the capacity losses from frequency reg-

\section{Acknowledgements}

This work was supported with funding from the Department of Energy Vehicle Technologies Office, under the Grid Modernization Lab Consortium. Further sup- 
port was provided by the Laboratory Directed Research \& Development program at Lawrence Berkeley National Laboratory, by the Director, Office of Science, of the U.S. Department of Energy under Contract No. DE-AC02-05CH11231.

The vehicle-to-grid simulator (V2G-Sim), which was developed and applied in this study. V2G-Sim provides a valuable research, development, and deployment platform for users to understand how different vehicles will perform for different drivers, and how different vehicles will interact with the electricity grid. Stakeholders benefiting from V2G-Sim include engineers, scientists, researchers, policy makers, analysts, and investors across the automotive industry, electricity grid industry, policy and regulatory sectors, and end users. More information is available at http://v2gsim.lbl.gov.

\section{References}

[1] J. H. Williams, A. DeBenedictis, R. Ghanadan, A. Mahone, J. Moore, W. R. Morrow, S. Price, M. S. Torn, The technology path to deep greenhouse gas emissions cuts by 2050: the pivotal role of electricity, Science 335 (6064) (2012) 53-59.

[2] S. Saxena, J. MacDonald, S. Moura, Charging ahead on the transition to electric vehicles with standard 120V wall outlets, Applied Energy 157 (2015) 720-728.

[3] S. B. Peterson, J. Apt, J. Whitacre, Lithium-ion battery cell degradation resulting from realistic vehicle and vehicle-to-grid utilization, Journal of Power Sources 195 (8) (2010) 2385-2392.

[4] W. Kempton, J. Tomić, Vehicle-to-grid power fundamentals: calculating capacity and net revenue, Journal of Power Sources 144 (1) (2005) 268-279.

[5] E. Sortomme, M. A. El-Sharkawi, Optimal scheduling of vehicle-to-grid energy and ancillary services, Smart Grid, IEEE Transactions on 3 (1) (2012) 351-359.

[6] A. Y. Saber, G. K. Venayagamoorthy, Resource scheduling under uncertainty in a smart grid with renewables and plug-in vehicles, Systems Journal, IEEE 6 (1) (2012) 103-109. 
[7] A. Schuller, C. M. Flath, S. Gottwalt, Quantifying load flexibility of electric vehicles for renewable energy integration, Applied Energy 151 (2015) 335-344.

[8] D. P. Birnie, Solar-to-vehicle (S2V) systems for powering commuters of the future, Journal of Power Sources 186 (2) (2009) 539-542.

[9] S. Saxena, C. Le Floch, J. MacDonald, S. Moura, Quantifying ev battery end-oflife through analysis of travel needs with vehicle powertrain models, Journal of Power Sources 282 (2015) 265-276.

[10] E. Wood, M. Alexander, T. H. Bradley, Investigation of battery end-of-life conditions for plug-in hybrid electric vehicles, Journal of Power Sources 196 (11) (2011) 5147-5154.

[11] J. Vetter, P. Novák, M. Wagner, C. Veit, K.-C. Möller, J. Besenhard, M. Winter, M. Wohlfahrt-Mehrens, C. Vogler, A. Hammouche, Ageing mechanisms in lithium-ion batteries, Journal of power sources 147 (1) (2005) 269-281.

[12] J. Purewal, J. Wang, J. Graetz, S. Soukiazian, H. Tataria, M. W. Verbrugge, Degradation of lithium ion batteries employing graphite negatives and nickelcobalt-manganese oxide+ spinel manganese oxide positives: Part 2, chemicalmechanical degradation model, Journal of Power Sources 272 (2014) 1154-1161.

[13] G. Ning, B. N. Popov, Cycle life modeling of lithium-ion batteries, Journal of The Electrochemical Society 151 (10) (2004) A1584-A1591.

[14] R. Spotnitz, Simulation of capacity fade in lithium-ion batteries, Journal of Power Sources 113 (1) (2003) 72-80.

[15] J. Christensen, J. Newman, Effect of anode film resistance on the charge/discharge capacity of a lithium-ion battery, Journal of the Electrochemical Society 150 (11) (2003) A1416-A1420.

505 [16] C. Zhou, K. Qian, M. Allan, W. Zhou, Modeling of the cost of EV battery wear due to V2G application in power systems, Energy Conversion, IEEE Transactions on 26 (4) (2011) 1041-1050. 
[17] J. D. Bishop, C. J. Axon, D. Bonilla, M. Tran, D. Banister, M. D. McCulloch, Evaluating the impact of V2G services on the degradation of batteries in PHEV and EV, Applied Energy 111 (2013) 206-218.

[18] M. Palacín, A. de Guibert, Why do batteries fail?, Science 351 (6273) (2016) 1253292.

[19] J. Wang, J. Purewal, P. Liu, J. Hicks-Garner, S. Soukazian, E. Sherman, A. Sorenson, L. Vu, H. Tataria, M. W. Verbrugge, Degradation of lithium ion batteries employing graphite negatives and nickel-cobalt-manganese oxide+ spinel manganese oxide positives: Part 1, aging mechanisms and life estimation, Journal of Power Sources 269 (2014) 937-948.

[20] J. Neubauer, E. Wood, Thru-life impacts of driver aggression, climate, cabin thermal management, and battery thermal management on battery electric vehicle utility, Journal of Power Sources 259 (2014) 262-275.

[21] S. Saxena, J. MacDonald, D. Black, S. Kiliccote, Quantifying the flexibility for electric vehicles to offer demand response to reduce grid impacts without compromising individual driver mobility needs, Tech. rep., SAE Technical Paper (2015).

[22] T. Markel, A. Meintz, K. Hardy, B. Chen, T. Bohn, Multi-lab EV smart grid integration requirements study,2015

[23] U.S. Department of Transportation, National Household Travel Survey, 2009, <http://nhts.ornl.gov>

[24] R. V. Gopal, A. P. Rousseau, System analysis using multiple expert tools, Tech. rep., SAE Technical Paper (2011).

[25] National centers for environmental information, <http://www. ncdc. noaa.gov/>

[26] National solar radiation data base, <http://rredc.nrel.gov/solar/ old_data/nsrdb/>. 
[27] W. Kempton, V. Udo, K. Huber, K. Komara, S. Letendre, S. Baker, D. Brunner, N. Pearre, A test of vehicle-to-grid (V2G) for energy storage and frequency regulation in the PJM system, <http://www1.udel.edu/V2G/resources/ test-v2g-in-pjm-jan09.pdf>, 2009.

[28] C. Le Floch, F. Belletti, S. Moura, Optimal charging of electric vehicles for load shaping: a dual splitting framework with explicit convergence bounds, Transportation Electrification, IEEE Transactions on, 2 (1) (2016) 190 - 199.

[29] P. Siano, Demand response and smart gridsa survey, Renewable and Sustainable Energy Reviews 30 (2014) 461-478. 